\title{
Perfil dos pacientes atendidos no ambulatório de cirurgia de cabeça e pescoço no Hospital das Clínicas (UFC-CE)
}

\section{Profile of patients treated in head and neck surgery at Clinical Hospital (UFC-CE)}

Roberto Esmeraldo Nogueira Barbosa ${ }^{1}$. Luis Alberto Albano Ferreira ${ }^{2}$. Márcio Ribeiro Studart da Fonseca ${ }^{2}$. Francisco Monteiro de Castro Junior².

1 Médico Cirurgião Geral e Residente de Cirurgia de Cabeça e Pescoço do Hospital Universitário Walter Cantídio, Fortaleza, Ceará, Brasil. 2 Cirurgião de Cabeça e Pescoço, Médico Assistente do Departamento de Cirurgia de Cabeça e Pescoço do Hospital Universitário Walter Cantídio, Fortaleza, Ceará, Brasil.

\section{RESUMO}

Introdução: O Programa de Residência Médica de Cirurgia de Cabeça e Pescoço do HUWC foi fundado em 2005, tornandose a primeira residência médica da especialidade no eixo norte-nordeste. Avaliar o perfil dos pacientes atendidos é sempre importante para melhor compreensão da especialidade. Objetivo: Analisar de forma descritiva o perfil de atendimentos realizados no ambulatório de cirurgia de cabeça e pescoço. Material: Foram analisados 6315 atendimentos ambulatoriais no período de março de 2012 a dezembro de 2014. Métodos: Revisão do prontuário de todos os pacientes atendidos. Análise descritiva de dados de atendimento ambulatorial. Resultados: Foram realizados 6315 atendimentos no período, com uma média de quase 16 pacientes por turno de ambulatório, sendo 1448 pacientes atendidos pela primeira vez na instituição. As patologias de tireóide foram responsáveis por mais de $50 \%$ dos atendimentos. Tivemos uma média de idade de 53,18 anos. Conclusão: O perfil das patologias vista no ambulatório no nosso Hospital Universitário é o mesmo de outros serviços de excelência. $\mathrm{O}$ estudo comprova que o ambulatório de cirurgia de cabeça e pescoço encontra-se apto a atender a formação do residente do serviço, levando-o a se familiarizar com as principais patologias da especialidade e tornando-o apto a adentrar o mercado de trabalho.

Palavras-chave: Ambulatório hospitalar. Cabeça. Pescoço.

\section{ABSTRACT}

Introdution: The Medical Residency Program of Head and Neck Surgery of the UHWC was founded in 2005, becoming the first in the north-northeast axis. Evaluate the profiles of patients is always important for a better understanding of the specialty. Materials: Were analyzed 6315outpatients' appointments from March 2012 to December 2014. Method: Review of medical records of all patients seen. Descriptive analysis of outpatient appointments. Results: Were conducted 6315visits in the period,with an average of nearly 16 patients per turn of clinic, where 1448 patients seen for the first time in the institution.Thyroid diseases were responsible for over $50 \%$ of cases. We had a mean age of 53.18 years. Conclusion: The profile of the pathologies seen in the outpatient clinic of our University Hospitalis the same as other excellent services. The study shows that the head and neck surgery clinic is able to meet the training of resident service, taking you to familiarize yourself with the main pathologies of the specialty.

Keywords: Outpatient clinic. Head. Neck.

Autor correspondente: Roberto Esmeraldo Nogueira Barbosa. Rua General Silva Júnior, 800, apto 302, Fortaleza, Ceará. CEP: Telefone: +55 85 99998-9173. E-mail: bebeto_esmeraldo@hotmail.com

Conflito de interesses: Não há qualquer conflito de interesses por parte de qualquer um dos autores.

Recebido em: 13 Mai 2015; Revisado em: 16 Jun 2015; Aceito em: 13 Jul 2015. 


\section{INTRODUÇÃO}

A Universidade Federal do Ceará (UFC) é uma instituição de ensino superior pública e gratuita do governo federal brasileiro, localizada no Estado do Ceará, foi fundada em 16 de dezembro de 1954. Em 2013, foi considerada uma das mais bem avaliadas instituições do país pelo ranking do Índice Geral dos Cursos (IGC) do Ministério da Educação, no qual obteve o conceito 4, que indica o status "bom", colocando-a como segunda instituição de ensino superior do Nordeste. ${ }^{1}$

De acordo com Center for World University Rankings (CWUR), em 2013, a Universidade figurou entre as mil melhores instituições de ensino superior do mundo, na $964^{\mathrm{a}}$ posição, sendo a $16^{\mathrm{a}}$ entre as dezoito universidades brasileiras presentes no ranking. ${ }^{2}$

A Faculdade de medicina tem 66 anos e foi fundada em 12 de maio de 1948.

Em 1914 foi estabelecido no Memorial Sloan Kettering Cancer Center o primeiro serviço especializado em cirurgia de cabeça e pescoço do mundo. Em 1954, o Dr. Hayes Martin, considerado o pai da moderna medicina de tumores de cabeça e pescoço, fundou a Sociedade de Cirurgiões de Cabeça e Pescoço, normatizou a especialidade e editou um livro da área. No Brasil, os primeiros serviços estabelecidos foram a Fundação Antônio Prudente - Hospital AC Camargo (1953), Hospital das Clínicas - FMUSP (1957) e o Instituto Nacional do Câncer - INCA (1957). A Sociedade Brasileira de Cirurgia de Cabeça e Pescoço foi fundada em 1967. No entanto, só em 1982 a cirurgia de cabeça e pescoço foi oficialmente aceita como especialidade médica pelo Conselho Federal de Medicina. ${ }^{3}$

A especialidade de cirurgia de cabeça e pescoço é relativamente jovem, comparada com outras mais tradicionais, como dermatologia ou ginecologia. Ela surgiu pela necessidade de juntar conhecimentos de diversas áreas, como cirurgia geral, oncologia, endocrinologia, otorrinolaringologia, entre outras, para diagnosticar um espectro patológico amplo e complexo de afecções craniocervicofaciais. ${ }^{4}$

O Programa de Residência Médica do Serviço de Cirurgia de Cabeça e Pescoço do Hospital Universitário Walter Cantídio foi fundado em 2005, tornando-se a primeira residência médica da especialidade no eixo norte-nordeste.

O objetivo deste trabalho é analisar de forma descritiva o perfil de atendimentos realizados no ambulatório de cirurgia de cabeça e pescoço. O conhecimento desse perfil de atendimento ambulatorial é fundamental no ajuste de políticas de saúde pública, assim como na melhoria e desenvolvimento de programas de treinamento médico especializado.

\section{MATERIAIS E MÉTODOS}

Foram incluídos neste estudo todos os pacientes atendidos no ambulatório de cirurgia de cabeça e pescoço pelos residentes do serviço de residência médica do Hospital Universitário
Walter Cantídio, no período de março de 2012 a dezembro de 2014.

Foram atendidos nesse período 6315 pacientes no ambulatório.

Os ambulatórios ocorriam em três turnos semanais, sendo realizados 2 ambulatórios de retorno (2 turnos) para acompanhamento de pacientes admitidos no setor e pacientes que se encontram em pós-operatório e 1 ambulatório ( 1 turno) para pacientes encaminhados de outros serviços para admissão em nosso setor ( $1^{\mathrm{a}}$ consulta).

Os ambulatórios ocorriam sob a supervisão de um cirurgião de cabeça e pescoço e os residentes do serviço, onde eram discutidos os pormenores de cada caso.

Analisados dados dos pacientes retrospectivamente através de análise de prontuários. Classificando-os quanto ao sexo, patologias, tipo de consulta (primeira ou retorno) e idade.

Aprovado pelo comitê de ética do Hospital Universitário Walter Cantídio (número CAAE - 43327215.8.0000.5045).

Dados foram analisados através do Microsoft excel versão 14.0 (Office 2010).

\section{RESULTADOS}

Foram analisados os prontuários de todos os pacientes atendidos no período de março de 2012 até dezembro de 2014. Total de 6315 atendimentos ambulatoriais na área de cirurgia de cabeça e pescoço do Hospital Universitário Walter Cantídio (HUWC).

A Média de idade desses pacientes foi de 53 anos, variando de 2 a 93 anos.

Houve uma maior predominância do sexo feminino, relação de 2,2 mulheres para cada homem.

Doenças da glândula tireóide foram as consultas ambulatoriais mais comuns do nosso serviço, representando mais de 50\% dos atendimentos. Em seguida temos as patologias relacionadas a pele $(13,3 \%)$, cavidade oral $(10,1 \%)$, laringe $(6,2 \%)$, glândulas salivares, dentre outras. (Figura 1).

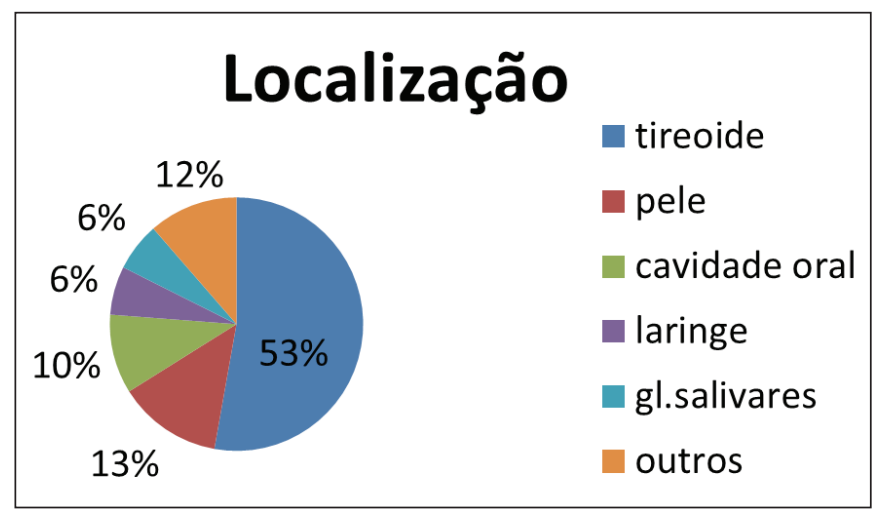

Figura 1. Localização das queixas atendidas no ambulatório. 
Existe uma predominância notória das patologias de tireóide em pacientes do sexo feminino. A Figura 2 mostra em números absolutos essas tendências.

\begin{tabular}{|c|c|c|c|c|c|c|c|}
\hline Localização & $\mathbf{N}$ & $\%$ & & & Localização & $n$ & $\%$ \\
\hline Tireóide & 293 & 8,8 & \multirow{6}{*}{ Homens } & \multirow{6}{*}{ Mulheres } & Tireóide & 3042 & 91,2 \\
\hline Pele & 473 & 56,3 & & & Pele & 367 & 43,7 \\
\hline Cav. oral & 419 & 62,6 & & & Cav. oral & 220 & 37,4 \\
\hline Laringe & 236 & 60,6 & & & Laringe & 153 & 39,4 \\
\hline Gl. salivar & 154 & 39,7 & & & Gl.salivar & 234 & 60,3 \\
\hline Massacervical & 143 & 58,3 & & & Massacervical & 102 & 41,7 \\
\hline Faringe & 88 & 68,8 & & & Faringe & 40 & 31,2 \\
\hline Tu partes moles & 71 & 55 & & & Tu partes moles & 58 & 45 \\
\hline Paratireóide & 28 & 28 & & & Paratireóide & 71 & 72 \\
\hline Tu ósseo & 45 & 51,7 & & & Tu ósseo & 42 & 48,3 \\
\hline Órbita & 15 & 41,7 & & & Órbita & 21 & 58,3 \\
\hline TOTAL & 1965 & 31 & & & TOTAL & 4350 & 69 \\
\hline
\end{tabular}

Figura 2. Prevalência das patologias por sexo.

Durante o período houve 398 atividades ambulatoriais, com uma média de 15 atendimentos por turno ambulatorial. Observa-se durante o período estudado um aumento na média de atendimentos ambulatoriais, de 12,5 atendimentos no primeiro período (março/2012 - fevereiro/2013) para 23,3 atendimentos no último período (março/2014 dezembro/2014).

Foram realizadas 1448 consultas de primeira vez, ou seja, admissão de pacientes no serviço. Esses pacientes chegam ao nosso serviço encaminhados pelos postos de saúde (assistência primária) ou referenciados por outras especialidades do próprio Hospital Universitário. Representaram quase 1/4 das consultas no período, sendo muito importante na formação do residente do serviço, pois através dessas consultas se capta novos casos para discussão e tratamento. Além disso, permitiu o acesso desses pacientes à assistência terciária qualificada.

A figura 3 ilustra essas consultas.

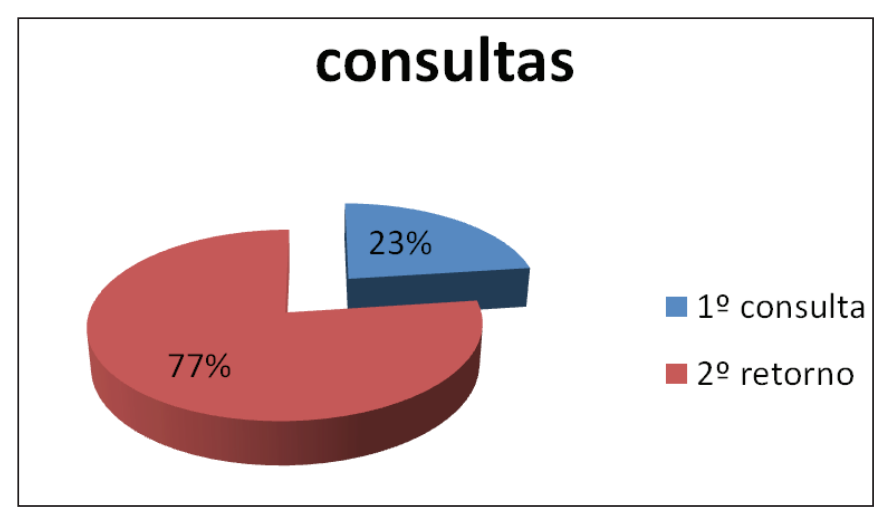

Figura 3. Consultas.

\section{DISCUSSÃO}

Os objetivos da educação ambulatorial são: reforço dos princípios da história e do exame físico; elaboração de avaliações custo efetivas; princípios de aconselhamento; desenvolvimento de uma base de conhecimentos de problemas gerais clínicos, cirúrgicos, psiquiátricos e neurológicos; reconhecimento das ramificações legais, sociais e econômicas de cada caso. ${ }^{5}$

O adequado conhecimento das características clínicas e epidemiológicas de pacientes é de interesse dos profissionais de saúde responsáveis pelo atendimento a estes doentes. ${ }^{6}$

Nas últimas décadas os serviços especializados em cirurgia de cabeça e pescoço tem se difundido tanto nas capitais como no interior do Brasil, acompanhando os processos de especialização crescente e de interiorização da medicina do país. Associado a isso observa-se também uma melhora na acessibilidade a métodos diagnósticos antes relativamente restritos. ${ }^{7}$ Neste cenário, talvez os fatores mais marcantes sejam o advento e difusão das estratégias de preservação de órgão e o aumento significativo na incidência do câncer de tireóide, assim como também o aumento da incidência de câncer de cabeça e pescoço no sexo feminino. Todas essas mudanças vêm causando alterações no perfil de atendimentos dos consultórios e ambulatórios dos cirurgiões de cabeça e pescoço. ${ }^{8}$

Sabendo-se que a especialidade de cirurgia de cabeça e pescoço trata principalmente as patologias tumorais, sejam elas benignas ou malignas, em nosso estudo foi observado uma média de idade de 53 anos, o que está de acordo com outros serviços de cabeça e pescoço que também relata média de idade entre a faixa etária de $51-65$ anos. ${ }^{9}$

Em relação ao gênero, os nossos corroboram com os já existentes na literatura que evidenciam maior incidência das patologias no sexo masculino, exceção essa se faz para as patologias de tireóide e glândulas salivares onde a predominância se mostra notória no sexo feminino. ${ }^{10}$ Particularmente em países em desenvolvimento, onde os homens são mais afetados do que as mulheres, embora nos últimos anos, tenha havido um aumento notável na incidência entre mulheres, que deve ser resultante da mudança dos hábitos tabagistas e etilistas. ${ }^{11}$

O fluxo ambulatorial de patologias da glândula tireóide, sejam elas malignas ou benignas, é o que predomina. Como comprovado na figura 1 , onde se observa que mais de $50 \%$ dos pacientes que realizaram consultas ambulatoriais são por patologias da glândula tireóide. Essas patologias tem uma predominância notória em pacientes do sexo feminino, ${ }^{12}$ corroborando com nosso estudo.

O Serviço do Hospital AC Camargo (Departamento de Cirurgia de Cabeça e Pescoço e Otorrinolaringologia) no ano de 2010 realizou admissão de 1692 pacientes. Quando colocamos a área de abrangência do serviço temos uma população que representa em números absolutos mais de quatro vezes a que compreende nossa área. Dados do IBGE 2014: população do estado de São Paulo 44.035.304 e a população do estado do Ceará (nossa área de abrangência) 8.842.791. Considerando isso podemos dizer que em termos proporcionais nossos números de admissões são compatíveis.

A nossa média de pacientes atendidos ambulatorialmente aumentou no decorrer dos períodos estudados, fato esse 
decorrente do fortalecimento do serviço no decorrer dos anos e a facilidade do paciente em chegar ao serviço terciário devido a melhoria no sistema básico nos últimos anos.

O CFM (Conselho Federal de Medicina) preconiza que se deve atender até 14 pacientes em um turno ambulatorial de 4 horas. Vemos que nossa média do último período encontra-se bem acima do número de atendimentos proposto pelo nosso conselho, 23,3 atendimentos por turno, contudo a média final chega próximo do ideal, 15,9 pacientes por ambulatório. ${ }^{13}$ Pelo CNRM (Conselho Nacional de Residência Médica) o residente em formação deve levar 30 minutos em uma consulta de primeira vez e 15 minutos em uma consulta de retorno. O que nos mostra que realizando as consultas dentro do tempo adequado o número de atendimentos será o que o CFM preconiza.

Nosso serviço contempla $30 \%$ da carga horária para parte ambulatorial, número o dobro do necessário segundo as diretrizes no CNRM. ${ }^{14}$

\section{REFERÊNCIAS}

1. Índice geral de cursos IGC [Internet]. Brasília: INEP; (C2011[acesso 2014 nov 20]. Disponível em: http://portal.inep.gov.br/educacaosuperior/indicadores/indice-geral-de-cursos-igc

2. UFC está entre as mil melhores universidades do mundo. O povo on line [Internet]. 2014 jul 31[acesso 2014 nov 20]; Fortaleza. Disponível em: http://www.opovo.com.br/app/fortaleza/2014/07/31/ noticiafortaleza,3290833/ufc-esta-entre-as-mil-melhoresuniversidades-do-mundo.shtml

3. Sociedade Brasileira de Cirurgia de cabeça e Pescoço [Internet]. São Paulo: SBCCP; C2012[acesso 2014 nov 20]. Disponível em: http://www.sbccp.org.br

4. Brandão LG, Araújo VJ Filho, Cernea CR. Manual do residente de cirurgia de cabeça e pescoço. 2 ed. São Paulo: Manole; 2013.

5. Hayashi SA, Hayden BB, Yager J, Guze PA. Graduate medical education in ambulatory care. Acad Med. 1989;64(10 supl):S16-21.

6. McCaffrey, TV. Evaluation of the thyroid nodule. Cancer control. 2000;7(3):223-8.

7. Jemal A, Thun M, Yu XQ, Hartman AM, Cokkinides V, Center $\mathrm{MM}$, et al. Changes in smoking prevalence among U.S. adults by state and region: estimates from the tobacco use supplement to the current population survey, 1992-2007. BMC Public Health. 2011;11:512.

8. Wang CJ, Knecht R. Current concepts of organ preservation in head and neck cancer. Eur Arch Otorhinolaryngol. 2011;268(4):4817.

\section{CONCLUSÕES}

O perfil das patologias vista no ambulatório no nosso Hospital Universitário é o mesmo de outros serviços de excelência.

O aumento da média ambulatorial no decorrer do período comprova a necessidade de mais um turno para atender a demanda de maneira organizada e didática para o residente.

Avaliar a criação de ambulatórios por patologia pode ser algo interessante, tornando as discussões mais adequadas. Dois turnos para patologias da tireóide e primeira consulta e dois turnos para as demais.

Por fim, o estudo comprova que o ambulatório de cirurgia de cabeça e pescoço encontra-se apto a atender a formação do residente do serviço, levando-o a se familiarizar com as principais patologias da especialidade e tornando-o apto a adentrar o mercado de trabalho.

9. Antunes AA, Antunes AP. Estudo retrospectivo e revisão de literatura dos tumores dos lábios: experiência de 28 anos. Revista Brasileira de Cancerologia. 2004;50(4):295-300.

10. Carvalho AL, Singh B, Spiro RH, Kowalski LP, Shah JP. Cancer of the oral cavity: a comparison between institutions in a developing and a developed nation. Head Neck. 2004;26(1):31-8.

11. Stewart BW, Kleihues P, editors. World cancer report. Lyon: IARC Press; 2003. 351 p.

12. Ward LS, Maciel RM. Câncer diferenciado da tireóide: fatores de risco e diagnóstico. São Paulo: AMB; 2006. (Projeto Diretrizes; v.5).

13. Santos AC. Número de pacientes a ser atendidos na jornada de trabalho do médico. Inexistência de normatização sobre o assunto. Parecer consulta $\mathrm{n}^{\circ}$ 009/2012 - CRM/PA - processo consulta $\mathrm{n}^{\circ}$ 437/2012, de 11 de junho de 2012 [Internet]. Belém, PA: Conselho Regional de Medicina; 2012 [acesso 2014 nov 20]. Disponível em: http://www.portalmedico.org.br/pareceres/CRMPA/ pareceres/2012/9_2012.pdf

14. Cunha GR Filho. Dispõe sobre os requisitos mínimos dos programas de Residência Médica das especialidades médicas e dá outras providências. Resolução CNRM 04/83. Publicada no DOU de 30 de dezembro de 1983. Brasília, DF: Comissão Nacional de Residência Médica; 1983 [acesso 2014 nov 20]. Disponível em: http://portal.mec.gov.br/sesu/arquivos/pdf/CNRM0483.pdf

\section{Como citar:}

Barbosa RE, Ferreira LA, Fonseca MR, Castro FM Junior. Perfil dos pacientes atendidos no ambulatório de cirurgia de cabeça e pescoço no Hospital das Clínicas (UFC-CE). Rev Med UFC. 2015 jul-dez;55(2):11-14. 